\title{
Wavelet Transform and Terahertz Local Tomography
}

\author{
Xiaoxia Yin ${ }^{a}$ and Brian W.-H. $\mathrm{Ng}^{a}$ and Bradley Ferguson ${ }^{a b}$ and Derek Abbott $^{a}$ \\ ${ }^{a}$ Centre for Biomedical Engineering and School of Electrical \& Electronic Engineering, The \\ University of Adelaide, SA 5005, Australia; \\ ${ }^{b}$ Tenix - Electronic Systems Division 2nd Avenue, Technology Park, Mawson Lakes, SA 5095, \\ Australia
}

\begin{abstract}
We use the theory of two dimensional discrete wavelet transforms to derive inversion formulas for the Radon transform of terahertz datasets. These inversion formulas with good localised properties are implemented for the reconstruction of terahertz imaging in the area of interest, with a significant reduction in the required measurements. As a form of optical coherent tomography, terahertz CT complements the current imaging techniques and offers a promising approach for achieving non-invasive inspection of solid materials, with potentially numerous applications in industrial manufacturing and biomedical engineering.
\end{abstract}

Keywords: Terahertz, T-rays, wavelet, computed tomography (CT), filtered back projection (FBP)

\section{INTRODUCTION}

Terahertz radiation (T-rays) is a collective term to describe the part of the electromagnetic spectrum from 0.1 THz to $10 \mathrm{THz} .{ }^{1-6}$ The application of THz time domain spectroscopy (THz-TDS), especially in the biomedical and security fields and in the fields of material science, is attractive owing to two intrinsic properties: a nonionising nature and the ability to penetrate dry, non-polar and non-metallic materials. ${ }^{7}$ Compared to traditional X-ray techniques, terahertz 3D imaging uses coherent tomography, which allows us to obtain both phase and amplitude information of an object.

This paper for CT reconstruction is motivated by terahertz TDS imaging mechanisms and focuses on terahertz CT imaging with reduced projection angles. The main goal of this paper is to present a wavelet based reconstruction algorithm for terahertz computed tomography and to show how this algorithm can be used to rapidly reconstruct the region of interest (ROI) with a reduction in the measurements of terahertz responses, compared with a standard reconstruction. The current algorithm provides new insight into the relationship between local reconstruction, local projection, and the resolution of terahertz coherent tomography. This algorithm is sensitive to terahertz data when reconstructing local projections using wavelet techniques, resulting in variations in the boundary of the local projection region after the wavelet transform, which gives rise to different resolution and reconstructed image sizes. This algorithm generates the approximation and detail images separately, and the final reconstruction is found by inverse wavelet transform. The algorithm reconstructs the area of interest via applying two sets of data from two different target experiments: polystyrene with hold inside and a tube inside a vial - a simple nested structure. For the first datasets, we reconstruct (i) a center region of 16 pixel radius in a $100 \times 100$ pixel image using $46 \%$ of full data; (ii) an off-centre region of radius 30 pixels in a $100 \times 100$ image using $66 \%$ of full data. For the second set of datasets, we reconstruct a center region of 6 pixel radius in a $100 \times 100$ pixel radius image using $59 \%$ of full data.

This paper consists of six sections. Section II introduces a terahertz functional imaging system and gives an overview of the nonlocality of the Radon transform - this is important because we review the difference between the conventional Radon transform reconstruction and our modified Radon transform for coherent tomography. In addition, following review of traditional Radon transform, this section summarize the basics of the wavelet transform, and a full-data reconstruction technique based on the wavelet transform is also involved. Section IV then discusses the implementation of this method, and in Section V, the tomographic results are presented.

Further author information: (Send correspondence to Derek Abbott)

Derek Abbott: E-mail: dabbott@eleceng.adelaide.edu.au, Telephone: +61-8-8303-5748

Novel Optical Instrumentation for Biomedical Applications III, edited by Christian D. Depeursinge Proc. of SPIE-OSA Biomedical Optics, SPIE Vol. 6631, 663113, @ 2007 SPIE-OSA · 1605-7422/07/\$18 


\section{METHODOLOGY}

\subsection{A Brief Introduction to Terahertz Imaging}

A terahertz CT system is based on a chirped terahertz time domain spectroscopy scanned imaging system. The target is mounted on a motion stage that allows it to be translated along the $x$ and $z$ axes. Meanwhile, the object can also be rotated and linearly moved. The detailed information for the chirped pulse scanning and relative rectangular coordinate system and polar coordinates for imaging reconstruction please see. ${ }^{8}$ The current research builds upon our previous work represented in Ferguson et al. ${ }^{8}$

\subsection{An Overview of CT and Terahertz CT}

Normally, a filtered back projection algorithm begins with a collection of sinograms obtained from projection measurements. A sinogram is simply generated via a collection of the projections at all the projection angles. It satisfies the following equation:

$$
s(\xi, \theta)=\int o(x, z) \mathrm{d} \xi
$$

where all points on projection offset $\xi$ satisfy the equation: $x \cos \theta+z \sin \theta=\xi$ and $o$ denotes the measured image intensity of a target object, which is a function of pixel position in an $\mathrm{x}$ and $\mathrm{z}$ plane.

The filtered back projection algorithm for terahertz CT reconstruction is expressed as follows:

$$
I(x, y)=\int_{0}^{\pi}\left[\int_{-\infty}^{\infty} S(\theta, \beta)|\beta| \exp [i 2 \pi \beta \xi] \mathrm{d} \beta\right] \mathrm{d} \theta
$$

where $S(\theta, \beta)$ is the spatial Fourier transform of the parallel projection data, defined as

$$
S(\theta, \beta)=\int_{-\infty}^{\infty} s(\theta, \xi) \exp [-i 2 \pi \beta \xi] \mathrm{d} \xi,
$$

here, $s(\theta, \xi)$ is the measured projection data, $\beta$ is the spatial frequency in the $\xi$ direction. It should be noted that the operation of the ramp filter $|\beta|$, as illustrated in Eq. (3), is equivalent to a differentiation followed by a Hilbert transform, which introduces a discontinuity in the derivative of the Fourier transform at zero frequency, while wavelet based local reconstruction, represented in this paper, ensures localised features of a local basis for image recovery.

\subsubsection{Calculation of Terahertz Parameters for Reconstruction of Terahertz CT}

One of the advantages that terahertz $\mathrm{CT}$ has over X-ray CT is that $s(\theta, \xi)$ may be one of several parameters derived from terahertz pulses. Fundamentally, a terahertz CT setup is capable of measuring the transmitted terahertz pulse as a function of time $t$, for a given projection angle and projection offset. In principle, terahertz sinograms can be obtained in both time and frequency domains:

\section{Frequency domain sinogram for terahertz CT:}

The measured terahertz pulse is a function of time $t$, at a given projection angle and projection offset $p_{d}(t, \theta, \xi)$. Let us denote the Fourier transform of this time domain pulse by $P_{d}(\omega, \theta, \xi)$. The reference pulse $p_{i}(t)$ and the corresponding Fourier response $P_{i}(\omega)$ can be measured by removing the target object from background. If the target is rotated and probed by terahertz beams, $P_{d}(\omega, \theta, \xi)$ may be evaluated by adding sufficient projection angles to allow the filtered back projection algorithm to be applied at each specific frequency $\omega$. This is based on the approximation that the detected terahertz signal is viewed as a linear integral of the incident terahertz pulse,

$$
P_{d}(\omega, \theta, \xi)=P_{i}(\omega) \exp \left[\int_{L(\theta, \xi)} \frac{-i \omega \hat{n}(r)}{c} \mathrm{~d} r\right]
$$

where $P_{d}$ and $P_{i}$ are the Fourier transforms of the detected and incident terahertz signals, respectively; $c$ is the speed of light in free space, $L$ is the projection path, a straight line between the source and detector. The unknown complex refractive index of the sample is denoted by $\hat{n}(\omega, r)=n_{\delta}(\omega, r)+i k(\omega, r)$, where $n_{\delta}(\omega, r)$ is 
the real refractive index deviation and $k(\omega, r)$ is the extinction coefficient, related to absorption coefficient $\alpha$ via $k(\omega, r)=\alpha / 2 k_{i}\left(k_{i}\right.$ is the incident extinction coefficient). Let us define that,

$$
\begin{aligned}
P_{n} \doteq\left[\frac{P_{d}(\theta, \xi)}{P_{i}(\theta, \xi)}\right] / k_{i}=\int_{L} n_{\delta}(r) d r=\Re\left\{n_{\delta}(r)\right\} \\
P_{\alpha} \doteq-2\left\|\frac{P_{d}(\theta, \xi)}{P_{i}(\theta, \xi)}\right\|=\int_{L} \alpha(r) d r=\Re\{\alpha(r)\}
\end{aligned}
$$

where $\arg (x)$ denotes the phase or argument of complex valued $x,\|x\|$ denotes the magnitude of the complex scalar $x$, and $P_{n}$ and $P_{\alpha}$ are the projection data inputs to the filtered back projection algorithm as required to reconstruct $n_{\delta}$ and $\alpha$, respectively, at a specific terahertz frequency $\omega$. The sign $r$ denotes the position of the incident field (the sensor). The frequency signogram is applied to the vial and tube data sets (see later) for this paper's experiments.

Time domain signogram for terahertz CT: One of the advantages that terahertz CT has over X-ray CT is that $s(\theta, \xi)$ may be one of several parameters derived from terahertz pulses. Fundamentally, a terahertz CT setup is capable of measuring the transmitted terahertz pulse as a function of time $t$, for a given projection angle and projection offset. In principle, terahertz sinograms can be obtained in both time and frequency domains. In this paper, we review the calculation of terahertz sinograms in the time domain.

This method is based on the assumption that the target is of less dispersion and therefore the $\mathrm{THz}$ pulse shape is less unchanged after propagation through the target apart from attenuation and time delay. A reference terahertz pulse $p_{i}(t)$ is measured without the target in place. To estimate the phase shift $t$ of a terahertz pulse $p_{d}(t)$, the two signals are resampled at a higher rate using low-pass interpolation. The two interpolated signals are then cross-correlated, and the maximised cross-correlation product at each angle as the lag is taken as the estimation of the phase delay of $p_{d}(t)$.

Timing sinogram can be calculated based on the following equation

$$
p_{\text {time }}=\int_{L(\theta, \xi)} T_{\text {delay }} d r
$$

here, $p_{\text {time }}$ denotes the sinogram image in the time domain, recovered from the maximum time delay. As for detailed information for the time domain signogram calculation, please refer to Ferguson et al. ${ }^{8}$

\subsection{Two Dimensional Wavelet Based CT Reconstruction}

\subsubsection{Two Dimensional Wavelet Transform (2D DWT)}

Wavelet transforms play an important role in many image processing algorithms. Fundamentally, wavelet decomposition corresponds to a multiresolution analysis of a signal. ${ }^{9-13}$ This has the advantage of much improved joint time-frequency localisation over Fourier based techniques. In practice, it is nearly always implemented using digital filters and downsamplers. In two dimensions, the discrete version of a wavelet transform can be realised by a $2 \mathrm{D}$ scaling function, $\phi(x, y)$, and three $2 \mathrm{D}$ wavelets, $\psi_{1}(x, y), \psi_{2}(x, y)$, and $\psi_{3}(x, y)$, which are calculated by taking the 1D wavelet transform along the rows of $f(x, y)$ and the resulting columns. The 2D scaling function and $2 \mathrm{D}$ wavelet functions satisfy the equations represented in Gonzalez and Woods. ${ }^{9}$

Our current experiment uses symmetric (linear phase) filters for the analysis of tomographic reconstruction. Let $h_{0}, h_{1}$, denote a pair of linear phase low- and high-pass wavelet filters and $\tilde{h}_{0}, \tilde{h}_{1}$ denote the corresponding reconstruction filters. The discrete approximation at resolution $2^{j}$ can be obtained by combination of the details and approximation at resolution $2^{j+1}$ using reconstructed wavelet filters:

$$
\begin{aligned}
c_{j}(k, l)= & \sum_{m, n} \tilde{h}_{0}(k-2 m) \tilde{h}_{0}(l-2 n) c_{j+1}(m, n)+\tilde{h}_{0}(k-2 m) \tilde{h}_{1}(l-2 n) d_{j+1}^{H}(m, n) \\
& +\tilde{h}_{1}(k-2 m) \tilde{h}_{0}(l-2 n) d_{j+1}^{V}(m, n)+\tilde{h}_{1}(k-2 m) \tilde{h}_{1}(l-2 n) d_{j+1}^{D}(m, n) .
\end{aligned}
$$

Our method is to focus the wavelet application on recovering local images from wavelet approximate and detail coefficients. In order to support the reconstructed filter for the recovery of the local target area, the calculation of these reconstructed coefficients includes the region of interest and a margin area. 


\subsubsection{Two Dimensional Wavelet Reconstruction}

This section briefly describes an algorithm, which is applied to obtain the wavelet coefficients of a function on $\mathbb{R}^{2}$ space, based on the measured terahertz projection data. This method enables reduced computation compared to the wavelet coefficients obtained, after conducting wavelet transforms in a reconstructed image. Moreover, the wavelet coefficients are calculated locally allowing the local reconstruction to yield local computed tomography. ${ }^{14}$ The main formulas for 2D DWT, on projection data, for the reconstruction of a CT image are introduced, which are realised via performing separate wavelet transforms on 1D projection data.

The filtered back projection algorithm for terahertz CT reconstruction is expressed as follows:

$$
I(x, y)=\int_{0}^{\pi}\left[\int_{-\infty}^{\infty} S(\theta, \beta)|\beta| G_{2^{j}}(\beta \cos \theta, \beta \sin \theta) \exp (i 2 \pi \beta \xi) \mathrm{d} \beta\right] \mathrm{d} \theta
$$

where $S(\theta, \beta)$ and $G_{2^{j}}\left(\beta_{1}, \beta_{2}\right)$ are the spatial Fourier transforms of $s(\theta, \xi)$ and $g_{2^{j}}$ (a wavelet ramp filter in the time domain), respectively.

The function enables image reconstruction as the conventional inversion of the Radon transform method, while the ramp filter $|\beta|$ is replaced by the wavelet ramp filter $|\beta| G_{2^{j}}(\beta \cos \theta, \beta \sin \theta)$.

As for a separable wavelet basis, the reconstructed approximate and detail coefficients are easily achieved via referring to Rashid-Farrokhi et al. ${ }^{14}$

For the current image reconstruction, only one $2 \mathrm{D}$ wavelet transform step is used. This is because the single level decomposition of scaling and wavelet ramp filters allows clear reconstruction of an image in the ROI and it avoids more computational complexity due to more levels of WT employed. ${ }^{14}$ The wavelet reconstruction formulas in Eq. (9) allow for such reconstruct by setting $j=1$. The 2D inversion of the traditional wavelet transform (IWT) is conducted on the back projection of reconstructed approximate and detail sinograms, after the decomposition procedure is performed.

\subsection{Local Reconstruction Using Wavelets}

A significant characteristic of the wavelet transform is its a large number of vanishing moments. Hilbert transforms of functions with many vanishing moments have been shown to decay very rapidly at infinity. ${ }^{15}$ In other words, a wavelet function with compactly supported allows a local basis to maintain its localised features after Hilbert transformation. ${ }^{15}$ Fig. (1)(a)-(c) illustrates the ramp filter over the full frequency domain, the BiorSplines bi-orthogonal scaling and wavelet filters and the ramp filtered version of the BiorSplines bi-orthogonal wavelet and scaling filters, where the $\mathrm{X}$ axis means the number of time or frequency samples, and $\mathrm{Y}$ axis means the relative amplitude. Fig. (1)(c) essentially shows the essentially compact support after applying Hilbert transforms. Therefore, the wavelet and scaling coefficients for some wavelet basis can be calculated after applying the projections passing through the region of interest plus a margin for the support of the wavelet and scaling ramp filters. These reconstructed coefficients, in this experiment, are then directly applied to the inverse wavelet transforms for terahertz image reconstruction.

\section{IMPLEMENTATION}

\subsection{Practical Consideration}

The current research based on terahertz imaging is most closely related to Rashid-Farrokhi et al. ${ }^{14}$ In this work, we experiment with the $2 \mathrm{D}$ wavelet technique using terahertz tomographic data by modifying the measured projections. As we show later, this modification involves an extrapolation technique to avoid edge effects due to sinogram truncation. It is observed that approximate coefficients of a scaling function shows good localised features in the local reconstruction using our algorithm, where the reconstructed intensity of an image varies much between different target materials. It should be noted that, in the application of terahertz data for local reconstruction, it is found that the intensity at the edges of the region of exposure (ROE) in terahertz projections, where nonlocal data is set to zero, varies considerably after conducting either a traditional ramp filter or scaling and wavelet ramp filters. 


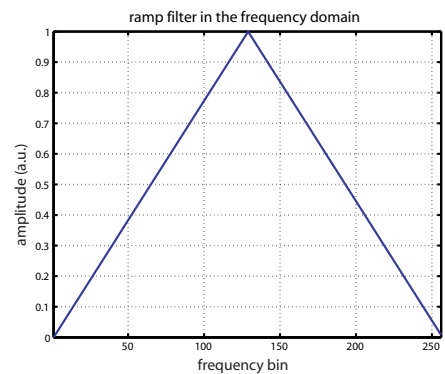

(a)

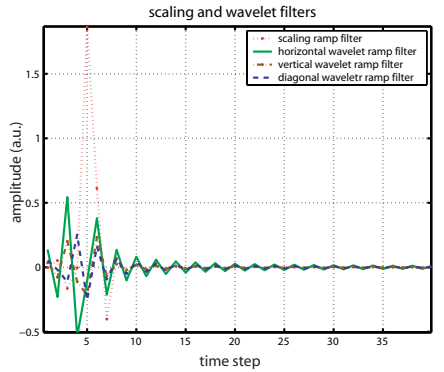

(b)

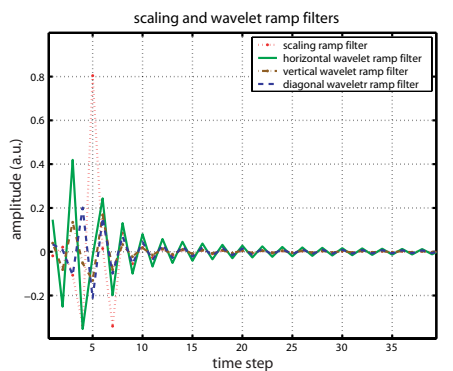

(c)

Figure 1. (a) Illustration of a traditional ramp filter. (b) and (c) Illustration of the scaling and wavelet ramp filters at the sixth projection angle (43.2 degree) using BiorSplines 2.2 wavelet, respectively.

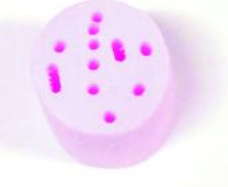

(a)

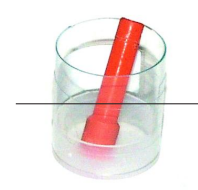

(b)

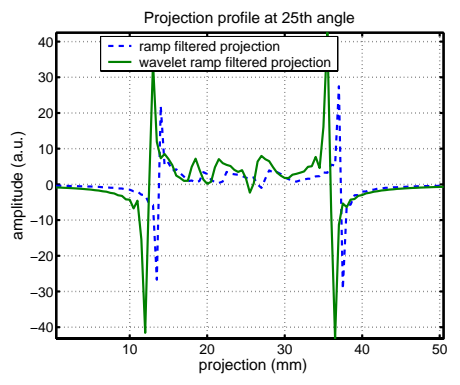

(c)

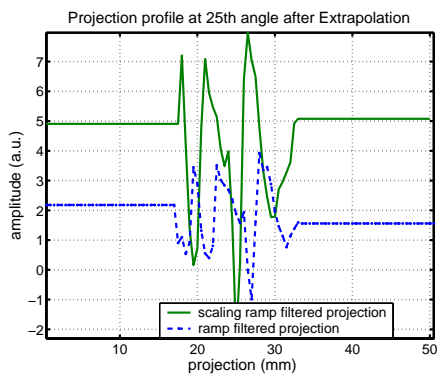

(d)

Figure 2. (a) An optical image of a target with $2 \mathrm{~mm}$ diameter holes drilled into a polystyrene cylinder with varying interhole distances. (b) Target object photograph with simple nested structure. The line indicates the measurement height of $7 \mathrm{~mm}$. (c) Projection filtered by a scaling ramp filter and a traditional ramp filter, respectively. (d) Projection extrapolation outside the ROI after filtered projections.

In local reconstruction, artifacts are common close to the boundary of the ROE, which can readily be observed in the application to terahertz CT data. It is possible that reconstruction after applying a constant linear extrapolation results in missing information. This situation is illustrated in subsection 4.1.3. In this paper, there are two sets of terahertz data considered for reconstruction: a cylinder with holes inside (see the target photo in Fig 2(a)) and a nested structure of a tube inside a vial. For the first set of terahertz data (the sample photo in Fig 2(b)), with 101 projections at each of 25 projection angles covering a $180^{\circ}$ projection area in a $100 \times 100$ image. The line in the photo indicates the measurement height of $7 \mathrm{~mm}$. Two situations are analyzed for this target sample: (i) an ROE of diameter 42 pixels at the center of the image and (ii) an ROE of diameter 67 pixels offcenter to the image. For the second set of terahertz measurements, with 51 projections at each of 36 projection angles covering a $360^{\circ}$ projection area in a $100 \times 100$ image, an ROE of diameter 18 pixels at the center of the image is explored. Each of dataset has a pixel interval of $0.5 \mathrm{~mm}$.

Fig 2(c) shows sharp variation along the borders of the ROE after applying wavelet ramp filters and ramp filter, respectively, on each of the 1D projections, which result in an image appearing relatively weakened intensity compared to a large constant bias that exists along the reconstructed edges in the region of interest. The constant extrapolation we use is given by Eq. 25 in Rashid-Farrokhi et al. ${ }^{14}$ In order to fit terahertz signals, the current algorithm replaces $r_{e}$ with $\left(r_{e}-r_{a}\right)$ to diminish the artificial effect along the edge of ROE, where $r_{a}$ is the radius 
of the region of artifacts (ROA) centered at the origin.

Fig. 2(d) shows the extrapolated projection at the 25 th projection angle after the application of a scaling ramp filters and a ramp filter. The extrapolated projection removes spikes at the edge of the ROE. The extrapolation algorithm is suitable to the reconstruction of an image at the off-center area. In order to recover the cross-sectional image in the region of interest, the values of the sinograms outside of the ROE are set to zero. The traditional filtered back projection formulas and wavelet based reconstruction are applied to the remaining projections, respectively for analysis and comparison. The original terahertz sinogram image for the current terahertz data can be calculated via applying Eq. (6).

\subsubsection{Example Three}

The third experiment is performed on a simple sample with a nested structure, a tube inserted in a vial. Eq. (7) is applied to perform a Radon transform on the measured terahertz projection data. Eq. (9) is used to reconstruct a local image using a scaling ramp filter, with $G_{2^{j}}$ being multiplied by a shape scaling factor $\lambda$. The Eq. (9) can be rewritten

$$
\begin{aligned}
I(x, y)= & \int_{0}^{\pi} \mathrm{d} \theta \cdot\left[\int_{-\infty}^{\infty} \hat{s}(\theta, \beta)|\beta| \hat{g}_{2^{j}}\right. \\
& \cdot[\beta(\cos \theta \cdot \lambda), \beta(\sin \theta \cdot \lambda)] \exp [i 2 \pi \beta \xi] \mathrm{d} \beta] .
\end{aligned}
$$

Fig 3(a) shows the wavelet ramp filtered projection at the first sampled frequency before a shape scaling factor is applied, where a large ' $\mathrm{S}$ ' shape scaling ramp filtered projection is observed. Fig 3(b) shows and almost flat border along the projection after applying a shape scaling factor of $1 / 3$.

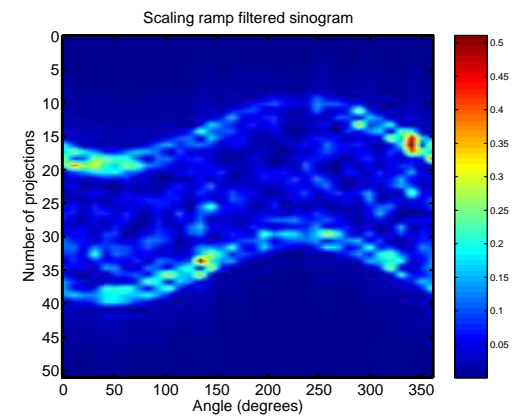

(a)

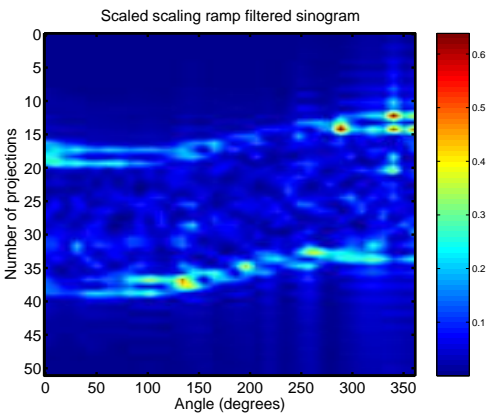

(b)

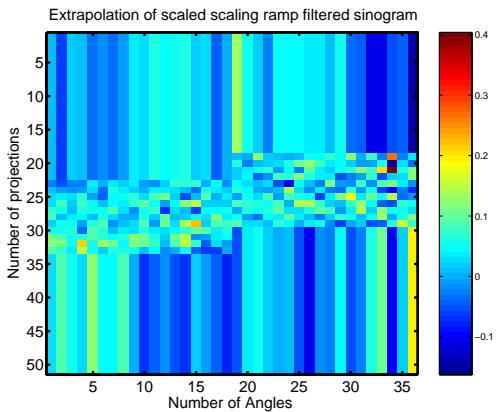

(c)

Figure 3. (a) Scaling ramp filtered projection before a shape scaling factor is applied. (b) Scaling wavelet ramp filtered projection after a shape scaling factor of $1 / 3$ is applied. (c) Illustration of the resultant signogram via extrapolation of scaling wavelet ramp filtered projection

In this example, it is observed that variation in the approximately flat border exists. In order to reduce the loss of necessary information in the ROI, different constants are adopted based on the different projection angles. Let us assume that the region of artifacts consists of two parts: $R O A_{1}$ and $R O A_{2}$ with the radii of $r_{a 1}$ and $r_{a 2}$, and with the projection angles of $\rho_{1} \in\left[0: \theta_{a 1}\right]$ and $\rho_{2} \in\left[0: \theta_{a 2}\right]$, respectively, in an image. To overcome the problem of edge discontinuities, truncated regions in the sinogram are extrapolated with a constant value, 
which satisfies Eq (11). Fig 3(c) shows the resultant sinogram via extrapolation of the scaling wavelet ramp filtered projection, with the same number of the projections at each projection angles being kept for convenience in calculation of reconstructed image.

$$
s_{\theta \text { local }}(p)=\left\{\begin{array}{l}
s_{\theta}(p), \ldots \\
\text { if } p \in\left(\mathrm{ROE}-R O A_{1}\right), \text { and } \theta \in\left[0: \theta_{a 1}\right] \\
s_{\theta}(p), \ldots \\
\text { if } p \in\left(\mathrm{ROE}-R O A_{2}\right), \text { and } \theta \in\left(\theta_{a 1}: \theta_{a 2}\right] \\
s_{\theta}\left(r \cos \left(\theta-\theta_{0}\right)+\left(r_{e}-r_{a 1}\right)\right), \ldots \\
\text { if } p \in\left[r \cos \left(\theta-\theta_{0}\right)+\left(r_{e}-r_{a 1}\right)\right], \ldots \\
\text { and } \theta \in\left[0: \theta_{a 1}\right] \\
s_{\theta}\left(r \cos \left(\theta-\theta_{0}\right)+\left(r_{e}-r_{a 2}\right)\right), \ldots \\
\text { if } p \in\left[r \cos \left(\theta-\theta_{0}\right)+\left(r_{e}-r_{a 2}\right)\right], \ldots \\
\text { and } \theta \in\left(\theta_{a 1}: \theta_{a 2}\right] \\
s_{\theta}\left(r \cos \left(\theta-\theta_{0}\right)-\left(r_{e}-r_{a 1}\right)\right), \ldots \\
\text { if } p \in\left[r \cos \left(\theta-\theta_{0}\right)-\left(r_{e}-r_{a 1}\right)\right], \ldots \\
\theta \in\left[0: \theta_{a 1}\right] \\
s_{\theta}\left(r \cos \left(\theta-\theta_{0}\right)-\left(r_{e}-r_{a 2}\right)\right), \ldots \\
\text { if } p \in\left[r \cos \left(\theta-\theta_{0}\right)-\left(r_{e}-r_{a 2}\right)\right], \ldots \\
\theta \in\left(\theta_{a 1}: \theta_{a 2}\right] .
\end{array}\right.
$$

\subsection{Algorithm Summary}

The wavelet based reconstruction algorithm assumes an image support of radius $R$, and the radius of the ROI is $r_{i}$. A radius $r_{e}=r_{i}+r_{a}$ is exposed, where $r_{a}$ is the extra margin with related to radius of ROA, which is produced by applying wavelet filters on the project data. The algorithm is summarized as follows.

\subsection{Algorithm Summary}

The wavelet based reconstruction algorithm assumes an image support of radius $R$, and the radius of the ROI is $r_{i}$. A radius $r_{e}=r_{i}+r_{a}$ is exposed, where $r_{a}$ is the extra margin with related to radius of ROA, which is produced by applying wavelet filters on the project data. The algorithm is summarized as follows.

1. The original projections are calculated from time or frequency parameters from terahertz measurements.

2. The region of exposure is truncated for the reconstruction of an image in the region of interest.

3. The region of exposure of each projection is filtered by modified wavelet filters at all projection angles. This step is to recover an image related to wavelet detailed coefficients.

4. The region of exposure of each projection is filtered by modified scaling filter at all projection angles, which will lead to the recovery of the approximation sub-image.

5. The projections from step 4 are extrapolated with constants to limit artifacts at the boundaries of the projections.

6. Filtered projections obtained in Step 3 and Step 4 are back projected to every other point to obtain the approximate and detail at the higher resolution. The remaining points are set to zero.

7. The image is reconstructed from the wavelet and scaling coefficients via a conventional inverse DWT. 


\section{RECONSTRUCTION RESULTS}

\subsection{Global Reconstruction}

A $196 \times 196$ pixel image of the polystyrene target is recovered from the wavelet and scaling coefficients using global data, shown in Fig. 4(a), without decomposition in the inverse wavelet transform for clarity and comparison. Each measured terahertz pulse is a function of time with 401 samples at uniform time intervals of 0.067 ps. Wavelet and scaling coefficients after back projection are shown in Fig. 4(b), where the BioSpline 2.2 biorthogonal basis is used. The quality of the reconstructed image is, as expected, almost indistinguishable from the reconstruction using traditional filtered back projection (FBP). The differences between the wavelet based reconstruction and traditional filtered back projection are evaluated using the reconstructed profiles at the 80th horizontal row of pixels and 80th vertical column of pixels, illustrated in Fig. 4(d) and (e), where it is not difficult to see the variation in detected hole positions using wavelet version of reconstruction (dash line) compared to traditional FBP algorithm (dash dot line).

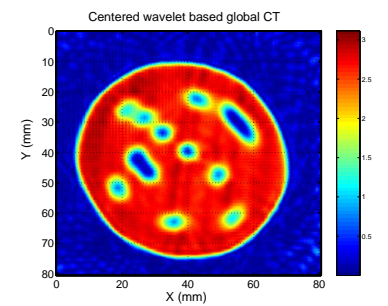

(a)
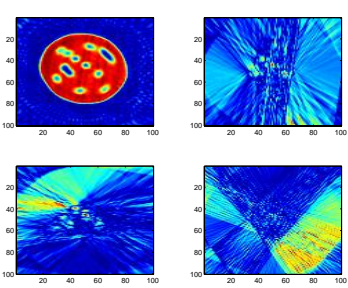

(b)

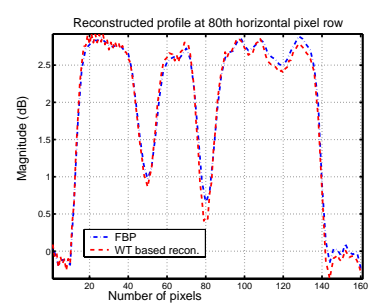

(c)

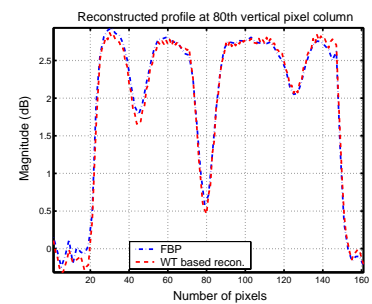

(d)

Figure 4. (a) A $196 \times 196$ pixel image of the polystyrene target is recovered from the wavelet and scaling coefficients using global data, without decomposition. (b) Wavelet and scaling coefficients after back projection. (c) Reconstructed profiles at the 80th horizontal pixel row. (d) Reconstructed profiles at the 80th vertical pixels column.

\subsection{Local Reconstruction at Center Area}

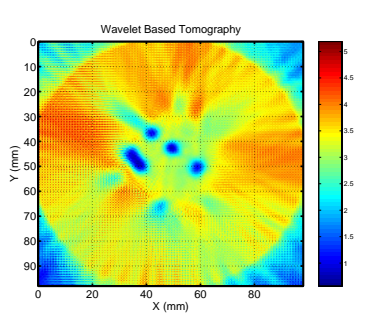

(a)

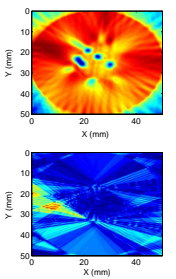

(b)

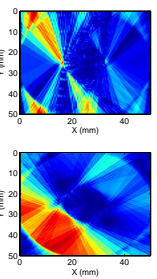

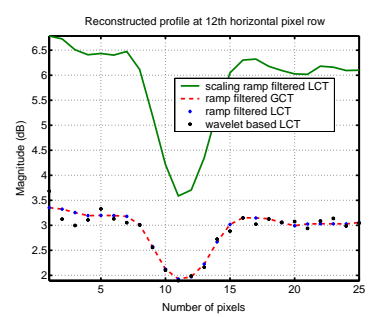

(c)

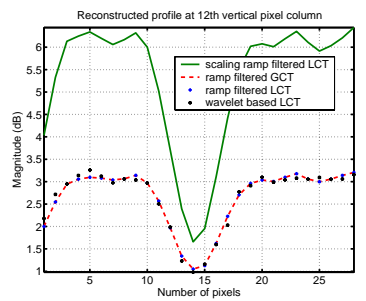

(d)

Figure 5. (a) Reconstructed image localised to a region of interest from the inverse wavelet transform. (b) Centered approximate and three detail reconstruction subimages along clockwise direction. (c) Reconstructed profiles at the 12th horizontal pixel row. (d) Reconstructed profiles at the 12 th vertical pixel column.

Fig. 5 shows reconstructed images centered at a radius of 16 pixels using the local reconstruction method outlined in Section4.1.1 and the traditional FBP algorithm. Each reconstruction is evaluated on a $100 \times 100$ image. Fig. 5(a) is the local reconstruction after extrapolation from wavelet and scaling filtered projection, with downsampling. Fig. 5(b) shows four subimages reconstructed from wavelet and scaling coefficients after constant 
extrapolation and BP. Fig. 5(c) and (d) shows the reconstruction profiles at the 12th horizontal row and vertical column of pixels corresponding to each reconstruction. As illustrated in Fig. 5(c) and (d), the profiles taken from the image reconstruction are scaled to improve construct.

\subsection{Local Reconstruction at off-Center Area}

Fig. 6(a)-(d) shows reconstructed images at an off-center area with a radius of 61 pixels using the current local reconstruction method and the traditional FBP algorithm. Each of the subfigures illustrates, for comparison, local reconstruction from extrapolated wavelet and scaling filtered projection after decomposition; the reconstruction of extrapolated approximate and detail coefficients after BP; the reconstruction profiles at the 28th horizontal row of pixels and the 12th vertical column of pixels are illustrated in Fig. 6(c) and (d), both of which correspond to the reconstructions from approximate wavelet coefficients, FBP based local and global recovery in the ROI. The reconstruction from wavelet approximate coefficients shows strong contrast in intensity for different media and FBP based local reconstruction shows a little higher intensity than FBP based global reconstruction.

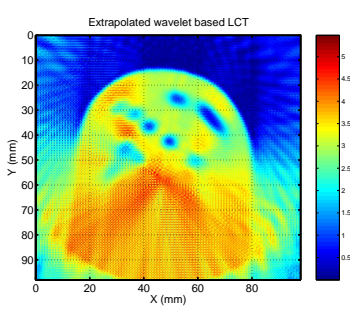

(a)

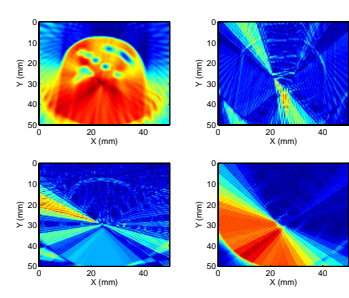

(b)

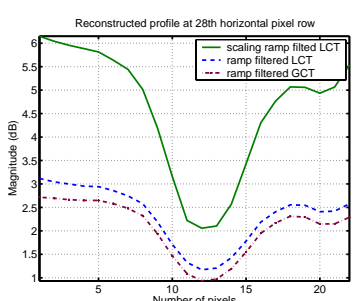

(c)

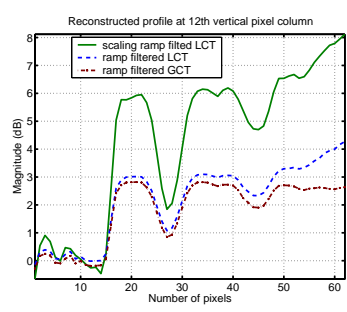

(d)

Figure 6. (a) A reconstructed image from the inverse wavelet transform without decomposition for clarity. (b) Offcentered approximate and three detail reconstructed subimages along clockwise direction. (c) Reconstructed profiles at the 28th horizontal row of pixels. (d) Reconstructed profiles at the 12 th vertical column of pixels.

\subsection{Example Three}

The nested structure of a tube inside a PET vial is imaged on a $100 \times 100$ grid. Its reconstruction from the wavelet and scaling coefficients using global data is shown in Fig. 7(a). The ten images span the sampled frequency scope from ten lowest frequencies, from $0.0213 \mathrm{THz}$ to $0.213 \mathrm{THz}$. Again, the BioSpline 2.2 biorthogonal basis is used. The quality of the reconstructed image is similar to using traditional filtered back projection (FBP), shown in Fig. 7(b), with a little increased recovered image intensity in the reconstructed subimages and a little discontinuity in the third reconstructed subimage compared to the traditional FBP algorithm.

Fig. 7(c) and (d) shows reconstructed images after extrapolation, evaluated on a $100 \times 100$ grid, at a center area with a disk radius of 6 pixels using the current local reconstruction method and the traditional FBP algorithm. They are enlarged for clarity. Each of the reconstructed subimages is illustrated, from $0.0213 \mathrm{THz}$ to $0.213 \mathrm{THz}$, relatively, with $59 \%$ of full projection data. The $59 \%$ of full projections is shown in Fig. $7(\mathrm{e})$ at the 6 th sampled frequency. The local reconstruction in the ROI from extrapolated wavelet and scaling filtered projection is shown in Fig. 7(c). Fig. 7(d) is the corresponding local reconstructions using FBP algorithm. The noise is obviously reduced in wavelet based reconstructed images, especially at the two frequencies of $0.0213 \mathrm{THz}$ and $0.0426 \mathrm{THz}$. It is valuable in the exploration of biomedical images using terahertz data, though a little aliasing occurs. The wavelet approximate and detailed coefficients after BP at the 7th sampled frequency is illustrated in Fig. 7(f), with a relative error of $29 \%$ from the approximate reconstruction.

\section{FUTURE WORK}

Since the current work involves only the one level of 2D DWT, it is interesting to explore the reconstruction algorithm with more levels of decomposition. Moreover, a research area of much current interest is the development 


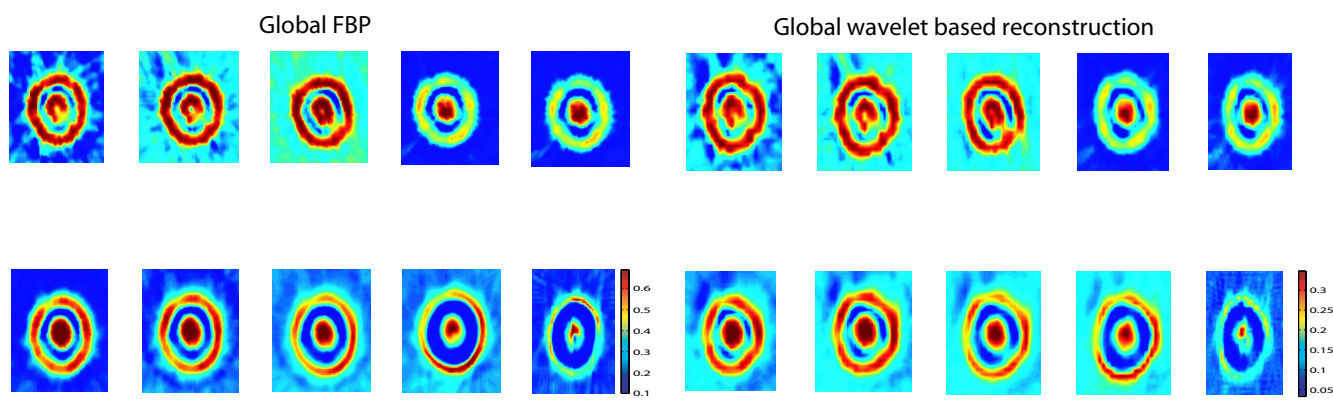

(a)
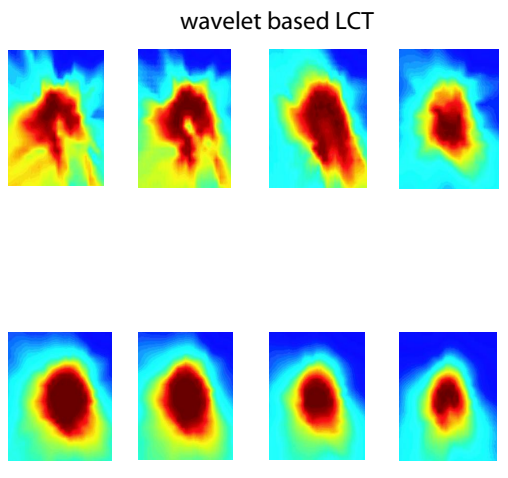

(c)

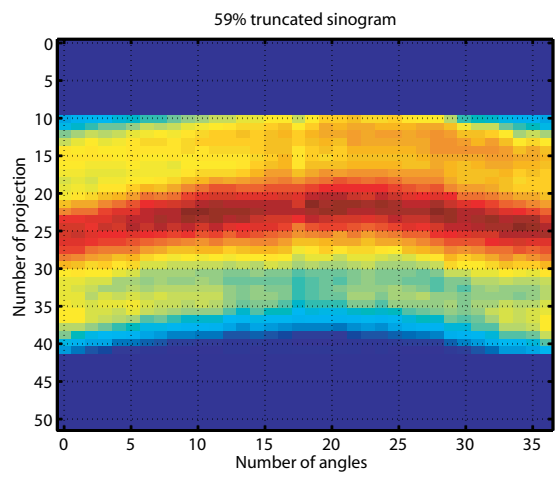

(e)
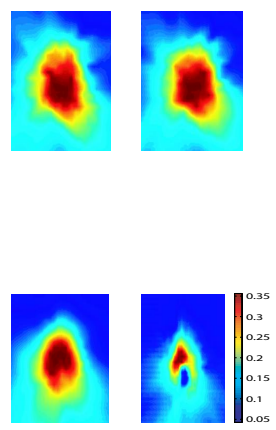
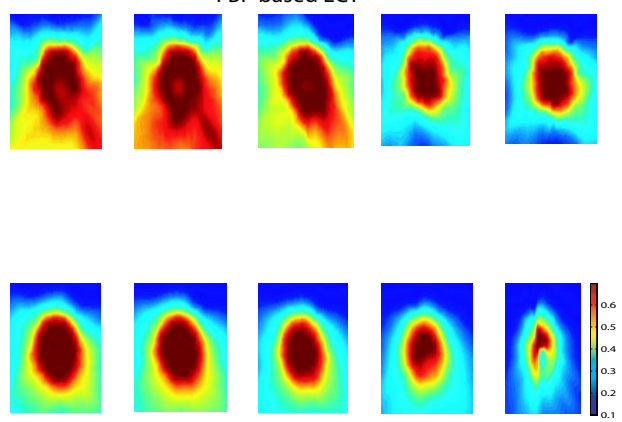

(d)
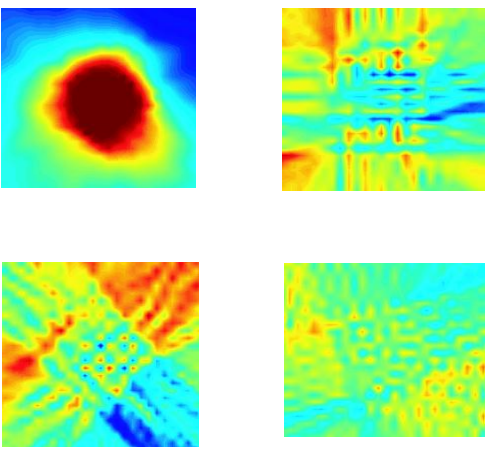

(f)

Figure 7. (a) Illustration of a $100 \times 100$ pixel global image of the tube inside a vial, with frequency range from $0.0213 \mathrm{THz}$ to $0.213 \mathrm{THz}$. It is recovered from the wavelet and scaling coefficients, after decomposition. (b) FBP based reconstruction from global measurement with size of $100 \times 100$ pixels and the same frequency range of (a). (c) A reconstructed image of the tube from the inverse wavelet transform after decompostion. (d) Corresponding reconstruction FBP algorithm using local projection data. (e) Illustration of the truncated projections with $59 \%$ of full data. (f) Approximate and detail reconstruction coefficients after BP using local projection data. 
of statistical based local tomography algorithm and techniques. ${ }^{16}$ It aims towards the actual localised reconstruction with relation to the terahertz measurement. The wavelet technique is critical for local reconstruction, and the relative wavelet transform coefficients can be thresholded to reduce the dimensions of the computational problem. ${ }^{17}$ In addition, the current resultant experiment relies on the fact that the Hilbert transform (part of the inverse RT) does not really change the compact support of scaling and wavelet functions. Selesnick in his paper $^{18}$ pointed out how to design coupled sets of scaling and wavelet functions which are approximate Hilbert transforms of each other. This could prove to be useful in the future work.

\section{CONCLUSION}

We have developed an algorithm to reconstruct the wavelet and scaling coefficients of a function from its signogram image of terahertz signals. Based on the observation that for some wavelet bases, with sufficient zero moments, the scaling and wavelet functions have essentially the same support after ramp filtering. Two targets are recovered from terahertz measurements, which demonstrates the current local reconstruction reconstruction method using a wavelet based transform scheme.

\section{REFERENCES}

1. K. L. Nguyen, M. L. Johns, L. Gladden, C. H. Worrall, P. Alexander, H. E. Beere, M. Pepper, D. A. Ritchie, J. Alton, S. Barbieri, and E. H. Linfield, "Three-dimensional imaging with a terahertz quantum cascade laser," Optics Express 14(6), pp. 2123-2129, 2006.

2. P. S. Carney, E. Wolf, and G. S. Agarwal, "Diffraction tomography using power extinction measurements," Journal of the Optical Society of America A: (Optics \& Vision) 16(11), pp. 2643-2648, 2002.

3. T. D. Dorney, W. W. Symes, R. G. Baraniuk, and D. M. Mittleman, "Terahertz multistatic reflection imaging," Journal of the Optical Society of America A 19, pp. 1432-1442, 2002.

4. S. Wang, B. Ferguson, and X.-C. Zhang, "Pulsed terahertz tomography," Journal of Physics D: Applied Physics 37, pp. R1-R36, (See also Erratum: Journal of Physics D : Applied Physics, 37, p. 964, 2004.), 2004.

5. A. B. Ruffin, J. Van Rudd, J. Decker, L. Sanchez-Palencia, L. Le Hors, J. F. Whitaker, and T. B. Norris, "Time reversal terahertz imaging," IEEE Journal of Quantum Electronics 38(8), pp. 1110-1119, 2002.

6. X.-X. Yin, B. W.-H. Ng, B. Ferguson, S. P. Mickan, and D. Abbott, "2-d wavelet segmentation in 3-d t-ray tomography," IEEE Sensors Journal 7(3), pp. 342-343, 2007.

7. B. Ferguson, S. Wang, D. Gray, D. Abbott, and X. Zhang, "Toward functional 3D T-ray imaging," Physics in Medicine and Biology (IOP) 47, pp. 3735-3742, 2002.

8. B. Ferguson, S. Wang, D. Gray, D. Abbott, and X. C. Zhang, "Identification of biological tissue using chirped probe THz imaging," Microelectronics Journal (Elsevier) 33(12), pp. 1043-1051, 2002.

9. R. C. Gonzalez and R. E. Woods, Digital Image Processing, Prentice-Hall, Inc., New Jersey, 2002.

10. S. Mallat, A Wavelet Tour of Signal Processing, Academic Press, San Diego, CA., 1999.

11. I. Daubechies, Ten Lectures on Wavelets, Society for Industrial and Applied Mathematics (SIAM), Philadelphia, PA, 1992.

12. A. Jensen and A. la Cour-Harbo, Ripples in Mathematics: The Discrete Wavelet Transform, Springer Verlag, Berlin, 2001.

13. D. Percival and A. Walden, Wavelet Methods for Time Series Analysis, Cambridge University Press, Cambridge, England, 2000.

14. F. Rashid-Farrokhi, K. Liu, C. Berenstein, and D. Walnut, "Wavelet-based multiresolution local tomography," IEEE Transactions on Image Processing 6(10), pp. 1412-1430, 1997.

15. A. H. Delaney and Y. Bresler, "Multiresolution tomographic reconstruction using wavelets," IEEE Transactions on Image Processing 4(6), pp. 799-813, 1995.

16. K. M. Hanson and G. W. Wecksung, "Bayesian estimation of 3-D objects from few radiographs," Journal of Optical Society of America 73(11), pp. 1501-1509, 1983.

17. A. Meyer-Baese, Pattern Recognition in Medical Imaging, Academic Press, Inc., Orlando, FL, USA, 2003.

18. I. W. Selesnick, "The design of approximate Hilbert transform pairs of wavelet bases," IEEE Transactions on Signal Processing 50(5), pp. 1144-1152, 2002. 